Revista Latinoamericana de la Papa 22 (2): 66 - 82

http://www.papaslatinas.org/revista.html

ISSN: 1853-4961

\title{
Nuevos cultivares de papa con resistencia a la rancha [Phytophthora infestans (Mont.) De Bary ] y adaptación al cambio climático
}

\author{
N. Zuñiga ${ }^{1 / *}$, M. Gastelo ${ }^{2}$. C. Bastos ${ }^{2}$, J. Reyes ${ }^{3}$. D. Alania ${ }^{3}$, E. Ninalaya ${ }^{4}$
}

Recibido: 02/10/2018

Aceptado: 30/11/2018

Accesible en línea: Diciembre de 2018

\section{Resumen}

El cultivo de papa en el Perú es la principal fuente de ingresos y en la alimentación de los pequeños productores de las zonas altoandinas. Para que los nuevos cultivares de papa sean adoptadas y difundidas luego de su liberación, es necesario aplicar metodologías como la selección varietal participativa (SVP), donde participan todos los actores de la cadena de valor del cultivo. Durante los años 2016-2017, se evaluaron tres clones de papa: CIP393079.4 (Flor Blanca), CIP387096.2 (Shulay), CIP396034.268 y los cultivares Única y Canchan, en ocho localidades de las regiones de Huánuco, Junín y Huancavelica en el Perú. La siembra de los experimentos fue en diseño de bloques completos al azar (DBCA) con tres repeticiones se realizó entre los meses de Setiembre a Noviembre del 2016 y la cosecha entre los meses de Marzo y Junio del 2017. Los criterios de selección fueron, a la floración: resistencia a la rancha, abundante follaje y tolerancia a la sequía; a la cosecha fueron: alto rendimiento, tamaño uniforme y sanidad de tubérculos. A la floración, los clones CIP396034.268 y CIP393079.4 fueron seleccionados por los agricultores. Los hombres seleccionaron además al clon CIP387096.2, mientras que las mujeres además de los dos primeros clones, seleccionaron al cultivar Canchan. A la cosecha los productores seleccionaron en las tres regiones a los clones CIP396034.268, CIP393079.4 y CIP387096.2 respectivamente. Los clones CIP387096.2, CIP393079.4 y CIP386034.268 fueron más resistentes a la rancha que los cultivares Canchan y Única en las regiones de Huancavelica, Huánuco y Junín.

Palabras clave adicionales: Clones, tubérculos, floración, cosecha, criterios.

New potato cultivars with resistance to late blight [Phytophthora infestans (Mont.) De Bary] and adaptation to climate change.

The cultivation of potatoes in Peru is the main source of income and food for small producers in the high Andean areas. For new potato cultivars to be adopted and disseminated after their release, it is necessary to apply methodologies such as participatory

\footnotetext{
* Autor para correspondencia: E mail: zunigaluz@yahoo.com

${ }^{1}$ Investigadora del Programa Nacional de Raíces y Tubérculos - INIA, Huancayo, Perú.

${ }^{2}$ Investigadores del Centro Internacional de la Papa en Lima y Huancayo.

3 Tesistas de la Universidad Nacional Hermilio Valdizan, Huánuco.

${ }^{4}$ Tesista de la Universidad Nacional del Centro del Perú.
} 
varietal selection (PVS), in which all the actors of the crop value chain participate. During the years 2016-2017, three potato clones were evaluated: CIP393079.4 (Flor Blanca), CIP387096.2 (Shulay), CIP396034.268 and the cultivars Única and Canchan, in eight locations in the regions of Huánuco, Junín and Huancavelica in Peru. The sowing of the experiments in randomized complete block design (DBA) with three repetitions was carried out between the months of September to November 2016 and the harvest between the months of March and June of 2017. The selection criteria were, at the flowering: resistance to late blight, abundant foliage and tolerance to drought; at harvest they were: high yield, uniform size and tubers health. At flowering, clones CIP396034.268 and CIP393079.4 were selected by the farmers. The men also selected the clone CIP387096.2, while the women, in addition to the first two clones, selected the cultivar Canchan. To the harvest, the producers selected clones CIP396034.268, CIP393079.4 and CIP387096.2 respectively in the three regions. Clones CIP387096.2, CIP393079.4 and CIP386034.268 were more resistant to late blight than the Canchan and Única cultivars in the regions of Huancavelica, Huánuco and Junín.

Additional keywords: Clones, tubers, flowering, harvest, criteria.

\section{Introducción}

El cultivo de papa se cultiva en 97,575 has de la sierra central del país, representando el $31 \%$ del área total sembrada en el Perú. Entre los principales problemas que presenta este cultivo son los factores bióticos y abióticos adversos para su producción, así entre los factores bióticos tenemos enfermedades como la rancha que si no es controlada adecuada y oportunamente puede causar la pérdida total del cultivo, y entre los abióticos las heladas, granizadas.

El cultivo de papa en el Perú es la principal fuente de ingresos $y$ en la alimentación de los pequeños productores de las zonas altoandinas. Para los habitantes de la ciudad es uno de los principales componentes de la canasta alimenticia y para los agentes de intermediación comercial es el principal producto por su volumen y valor de venta (Egúsquiza, 2000).

La obtención de nuevas cultivares mejoradas es muy importante para cumplir con estos fines, pero los cultivares de papa liberadas por los centros de investigación en el Perú en los últimos años han sido poco aceptadas por

los agricultores y su difusión ha sido lenta. Varios estudios muestran que el enfoque principal en los trabajos de selección de cultivares es preocuparse principalmente por aspectos agronómicos tales como altos rendimientos, resistencia a plagas y enfermedades, precocidad, etc. Sin embargo, estas características reflejan frecuentemente las prioridades establecidas por los investigadores, y no integran otras preferencias claves de los actores de la cadena alimentaria, que incluyen aspectos de consumo y de mercado tales como tener una papa de mejor sabor, con un color preferido, mejor precio, etc. (Cuesta et al., 2001; Gabriel et al., 2002 y Pardavé, 2004).

Para que los nuevos cultivares de papa sean fácilmente adoptadas y difundidas luego de su liberación, es necesario aplicar metodologías especiales durante el proceso de evaluación y selección, una de estas metodologías es la selección varietal participativa (SVP), donde participan todos los actores de la cadena de valor del 
cultivo: agricultores, técnicos, investigadores, instituciones y empresas públicas y privadas, en dos etapas fenológicas del cultivo; a la floración y a la cosecha, donde ellos participan activamente en dos actividades: Identificación y priorización de los criterios para seleccionar una nueva variedad y la evaluación de los genotipos candidatos para nuevos cultivares. Además se tendrá en cuenta el aspecto de equidad de género y así tener en cuenta las opiniones por separado de hombres y mujeres, muy importante para realizar una adecuada selección de las nuevas cultivares que permitirán sean adoptadas y difundidas más fácilmente luego de su liberación como producto del SVP.

En los últimos años se está incrementando los efectos del cambio climático, con la elevación de las temperaturas, distribución errática de las lluvias, esto ocasionaría el incremento de la presión de las enfermedades como la rancha, la presencia de épocas de sequía. Una manera de enfrentar estos factores adversos es mediante el uso de nuevos cultivares de papa con resistencia genética a la rancha, tolerancia a las heladas, sequia, calor etc. En este estudio el objetivo principal fue identificar nuevos cultivares y/o genotipos con resistencia o tolerancia a los efectos del cambio climático aplicando la metodología de selección varietal participativa, que nos permitan obtener nuevos cultivares de papa con rendimientos económicamente aceptables para asegurar su rentabilidad y la seguridad alimentaria, mejorando las condiciones de vida de los agricultores de las zonas altoandinas.

\section{Materiales y Métodos}

Durante los años 2016 -2017, se evaluaron tres clones de papa: CIP393079.4 (Flor Blanca), CIP387096.2 (Shulay), CIP396034.268 y los cultivares Única y Canchan (Tabla 1), en ocho localidades de las regiones de Huánuco, Junín y Huancavelica en el Perú (Tabla 2). Las siembras de los experimentos se realizaron entre los meses de Setiembre a noviembre del 2016 y la cosecha entre los meses de Marzo y Junio del 2017.

Tabla 1. Clones y cultivares en estudio.

\begin{tabular}{llllll}
\hline Clon/Variedad & Genealogìa & $\begin{array}{l}\text { Color } \\
\text { de Piel }\end{array}$ & $\begin{array}{l}\text { Color } \\
\text { de } \\
\text { Pulpa }\end{array}$ & $\begin{array}{l}\text { Forma } \\
\text { de } \\
\text { tubérculo }\end{array}$ & $\begin{array}{l}\text { Profundidad } \\
\text { de Ojos }\end{array}$ \\
\hline CIP387096.2 & CIP382119.17 X 575049 & Crema & Crema & Oval & Superficial \\
CIP393079.4 & CIP387004.13 x CIP390357.4 & Crema & Crema & Oval & Superficial \\
CIP396034.268 & CIP393042.50xCIP393280.64 & Rojo & Crema & Oblongo & Superficial \\
CANCHAN & & Rojo & Crema & Redondo & Intermedio \\
UNICA & & Rojo & Crema & Oblongo & Superficial \\
\hline
\end{tabular}


Tabla 2. Localidades donde se están conduciendo los experimentos.

\begin{tabular}{lllllc}
\hline$\#$ & Localidad & Distrito & Provincia & Región & Altitud msnm \\
\hline 1 & Checche & Pampas & Tayacaja & Huancavelica & 3300 \\
2 & Churcampa & Churcampa & Churcampa & Huancavelica & 3262 \\
3 & Santa Ana & El Tambo & Huancayo & Junín & 3270 \\
4 & Paca & Paca & Jauja & Junín & 3430 \\
5 & Huasahuasi & Huasahuasi & Tarma & Junín & 3341 \\
6 & Huarisca & Chupaca & Chupaca & Junín & 3331 \\
7 & Quechualoma & Churubamba & Huanuco & Huánuco & 2246 \\
8 & Mayobamba & Chinchao & Huánuco & Huánuco & 2934 \\
\hline
\end{tabular}

Se usó el diseño estadístico de Bloques Completos al Azar (BCA) con tres repeticiones de 30 plantas cada una, cada parcela estuvo compuesta de 6 surcos de $9.0 \mathrm{~m}$. de largo, de las cuales se cosecharon los dos surcos centrales para eliminar el efecto del borde. El área total cosechada de una parcela fue de $18.00 \mathrm{~m}^{2}$. El distanciamiento entre surcos fue de $1.00 \mathrm{~m}$ y entre plantas de $0.30 \mathrm{~m}$.

Se usó la metodología de Selección Varietal Participativa (SVP). Participan todos los actores de la cadena de valor del cultivo: agricultores, técnicos, investigadores, instituciones y empresas públicas y privadas de la región. La SVP se aplicó durante dos fases del experimento: en floración y cosecha. En cada una de estas fases se realizaron dos actividades: La primera fue la identificación y priorización de los criterios para seleccionar un nuevo cultivar y la segunda la evaluación de los genotipos candidatos para nuevos cultivares, identificándose los tres mejores genotipos de acuerdo con su apreciación visual y considerando los criterios priorizados. En la cosecha además se realizó la prueba organoléptica de los clones en evaluación para determinar su apariencia, sabor y textura. Se tomó los criterios y selección por separado para hombres y mujeres, muy importante para realizar una adecuada selección de las nuevas cultivares que permitirán sean adoptadas y difundidas más fácilmente luego de su liberación como producto del SVP.

Las variables que se tomaron fueron de dos tipos: variables cuantitativas $\mathrm{y}$ cualitativas. Entre las variables cualitativas se tomaron los criterios de selección a la floración y la cosecha, donde los participantes de la SVP mencionaron en forma oral las características más importantes para liberar una nueva variedad de papa teniendo en cuenta la etapa fenológica del cultivo, luego se procedió a priorizar estos criterios mediante elección tanto por hombres y mujeres. Para diferenciar los votos, a los hombres se les entrego semillas de maíz y a las mujeres se les entrego semillas de habas. Cada uno identifico el criterio más importante con tres semillas, el segundo con dos y el tercero con una semilla. Con la información obtenida se tabularon las preferencias por separado para hombres y mujeres. Luego se tomó el ranking de los 
clones en las dos fases del cultivo, para lo cual los participantes, primero hacían un recorrido por el experimento, luego emitían su calificación en cada repetición, escogiendo en forma individual y secreta los tres mejores clones. Con la información obtenida se tabularon los rankings de las preferencias tanto por hombres como por mujeres. Finalmente, dentro de la SVP a la cosecha se realizó la prueba organoléptica para determinar el sabor, textura y apariencia de los clones y cultivares en estudio en cada una de las localidades.

Entre las variables cuantitativas se tomó el daño por la rancha en la parte aérea de las plantas (hojas, tallos, flores y bayas), se realizaron de 4 a 6 evaluaciones, con intervalos de 15 días, tomándose el porcentaje de daño por la rancha en cada parcela experimental. Con esta información se calculó el área debajo de la curva de progreso de la enfermedad (AUDPC), valor que nos permitirá comparar la resistencia de los clones en estudio a través de las diferentes localidades, se realizó análisis de variancia simple para estudiar el efecto de la Rancha en cada localidad y combinado en cada Región. La comparación de medias de los clones se realizó usando la prueba de Waller/Duncan al $0.05 \%$. Para determinar el rendimiento se tomó el número y peso de tubérculos cosechados, contando y pesando los tubérculos que fueron clasificados en Extra, primera, segunda, tercera y cuarta, en los dos surcos centrales para evitar los efectos de borde. Con esta información se calculó el rendimiento de tubérculos comerciales y totales en toneladas por ha para cada localidad en las tres regiones. Se realizaron análisis de variancia simples para cada localidad y combinado para cada región, para el rendimiento comercial y total por hectárea. Para la comparación de medias se usó la prueba de Waller/Duncan al $\alpha=0.05$ Los análisis estadísticos se realizaron usando el programa SAS para Windows 9.4, SAS Inc.

\section{Resultados y Discusión}

Los criterios más importantes identificados en la SVP, en las regiones de Huánuco, Huancavelica y Junín fueron: A la floración, se identificó que los nuevos cultivares mejorados de papa, deben tener resistencia a la Rancha en primer lugar, en segundo lugar las plantas deben tener abundante follaje. Otros criterios fueron plantas con tallos gruesos, plantas grandes y plantas y que tengan tolerancia a la sequía. Estos criterios mencionados por los agricultores son muy importantes en la actualidad debido a que por efectos del cambio climático, la presencia de enfermedades como la rancha se incrementara e incluso se presentara en zonas donde antes no era un factor que afectaba al cultivo de papa a más de 4000 msnm, la necesidad de tener plantas con abundante follaje, está relacionado con la obtención de una mayor producción y los otros criterios están relacionados con la tolerancia a sequía, porque los productores están viendo que el régimen de lluvias es anormal en los últimos años y por ello están tratando de prevenir efectos negativos. Esta información coincide con información obtenida en otros ambientes dentro y fuera del país, donde los agricultores desean tener en el futuro, cultivares con resistencia a la rancha y tolerantes a la sequía, así cuando las precipitaciones son abundantes y la presión de la rancha se incrementa, las cultivares expresaran su resistencia y en épocas de escases de lluvias, se deberá expresar la tolerancia a sequía (Figura 1). 
Revista Latinoamericana de la Papa 22 (2): 66 - 82

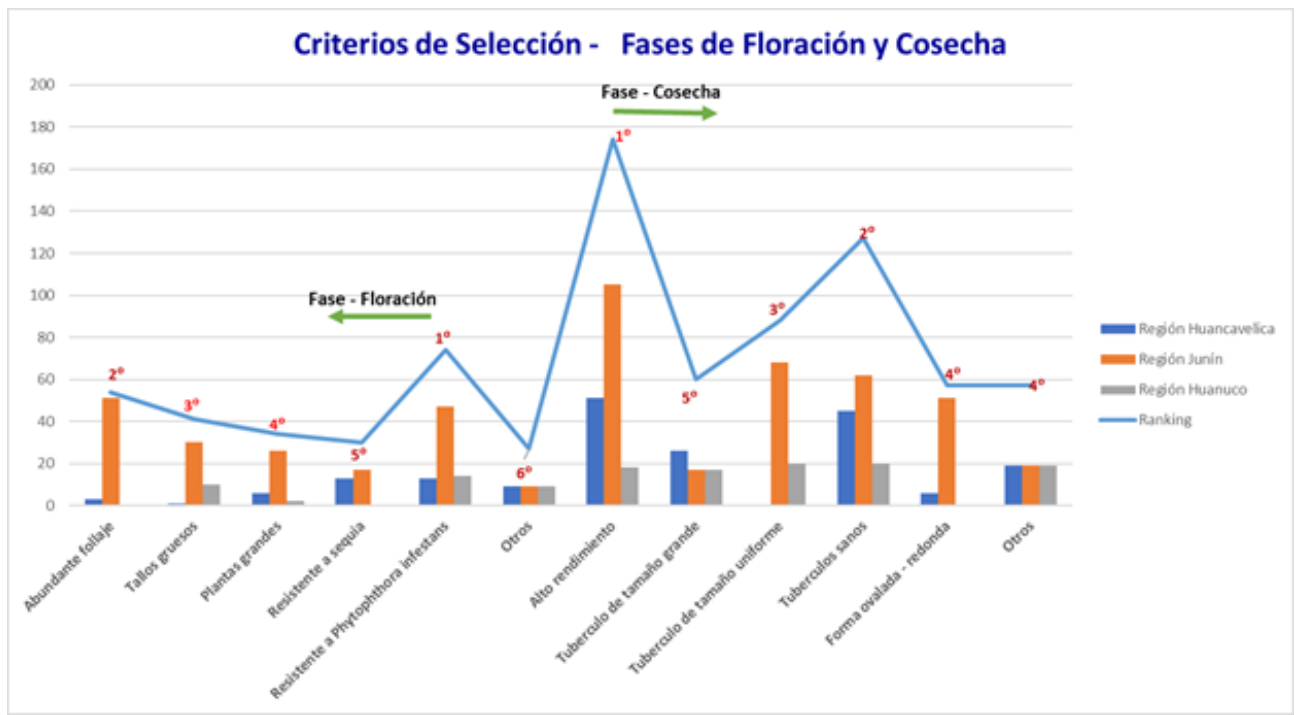

Figura 1. Criterios de selección a la floración y Cosecha durante las evaluaciones de SVP, en las regiones de Huánuco, Huancavelica y Junín. 2106-2017.

A la cosecha, los criterios más importantes de las tres regiones fueron: En primer lugar Alto rendimiento, seguido por tubérculos uniformes y sanos, estos criterios tienen estrecha relación con el mercado que es el objetivo final de los productores para generar ingresos y rentabilidad.

Disgregando los criterios por Región, a la floración, en todas las regiones el principal criterio es resistencia a la rancha, pero en Huancavelica además quieren tolerancia a la sequía y plantas grandes, esto está relacionado con la distribución errática de las lluvias en esta región y el tamaño grande de las plantas se asocia con el tamaño de las plantas de las cultivares nativas que predominan en esta zona. En Huánuco necesitan plantas vigorosas, que está relacionado con el deseo de tener alta producción de tubérculos para el mercado. En Junín los criterios son muy similares a la región Huánuco. Los criterios a la cosecha en cada una de las tres regiones son muy similares: Alto rendimiento de tubérculos en primer lugar, luego, tubérculos de tamaño grande, pero en Huancavelica los tubérculos deben ser grandes y en Huánuco los tubérculos deben ser sanos, relacionado a la alta presión de rancha en esta zona donde además es muy importante que esta enfermedad no afecte la calidad de los tubérculos.

Los clones CIP396034.268 y CIP393079.4 fueron los que ocuparon el primer y segundo lugar del ranking de preferencias por los agricultores en promedio de las tres regiones a la floración, seguida del cultivar Canchan. Disgregando por género, los hombres seleccionaron a la floración los clones CIP396034.268, CIP393079.4 y CIP387096.2 en primer, segundo y tercer lugar respectivamente; las mujeres seleccionaron los dos primeros clones también y además el cultivar Canchan, esto probablemente a que asocian la calidad de este cultivar para consumo en fresco y para fritura.

A la cosecha, en promedio de las tres regiones se seleccionaron los clones 
CIP396034.268, CIP393079.4 y CIP387096.2 ocupando el primer, segundo y tercer lugar del orden de mérito. Los hombres y las mujeres seleccionaron los mismos clones pero en diferente orden de mérito como se aprecia en la tabla 3. La selección de estos clones por los productores probablemente sea por que han observado la resistencia a la rancha que poseen estos clones en comparación a los cultivares Canchan y Ubica que son susceptibles a esta enfermedad y por la cantidad y calidad de la producción.

Tabla 3. Ranking de los clones y cultivares durante la SVP, en las regiones de Huánuco, Huancavelica y Junín a la Floración y Cosecha. 2016 -2017

\begin{tabular}{|c|c|c|c|c|c|c|c|c|c|c|c|c|c|c|c|c|c|c|}
\hline \multirow[b]{2}{*}{ Clones } & \multicolumn{4}{|c|}{ Huancavelica } & \multicolumn{4}{|c|}{ Junín } & \multicolumn{4}{|c|}{ Huanuco } & \multicolumn{3}{|c|}{ Total } & \multicolumn{3}{|c|}{ Ranking } \\
\hline & $\mathbf{H}$ & $\mathbf{M}$ & $\mathbf{T}$ & $\mathbf{R}$ & $\mathbf{H}$ & M & $\mathbf{T}$ & $\mathbf{R}$ & $\mathbf{H}$ & M & $\mathbf{T}$ & $\mathbf{R}$ & $\mathbf{H}+\mathbf{M}$ & $\mathbf{H}$ & $\mathbf{M}$ & $\mathbf{H}+\mathbf{M}$ & $\mathbf{H}$ & $\mathbf{M}$ \\
\hline \multicolumn{19}{|l|}{ Floración } \\
\hline CIP396034.268 & 49 & 40 & 89 & 3 & 144 & 289 & 433 & 2 & 90 & 21 & 111 & 2 & 633 & 283 & 350 & 1 & 1 & 2 \\
\hline UNICA & 58 & 73 & 131 & 1 & 60 & 95 & 155 & 5 & 26 & 20 & 46 & 4 & 332 & 144 & 188 & 5 & 5 & 5 \\
\hline Canchan & 49 & 67 & 116 & 2 & 92 & 194 & 286 & 3 & 18 & 15 & 33 & 5 & 435 & 159 & 276 & 3 & 4 & 3 \\
\hline CIP387096.2 & 22 & 32 & 54 & 5 & 78 & 137 & 215 & 4 & 91 & 36 & 127 & 1 & 396 & 191 & 205 & 4 & 3 & 4 \\
\hline CIP393079.4 & 35 & 44 & 79 & 4 & 155 & 293 & 448 & 1 & 64 & 21 & 85 & 3 & 612 & 254 & 358 & 2 & 2 & 1 \\
\hline \multicolumn{19}{|l|}{ Cosecha } \\
\hline CIP396034.268 & 91 & 32 & 123 & 2 & 186 & 244 & 430 & 1 & 59 & 48 & 107 & 4 & 660 & 336 & 324 & 1 & 2 & 1 \\
\hline UNICA & 74 & 33 & 107 & 3 & 112 & 172 & 284 & 4 & 93 & 27 & 120 & 3 & 511 & 279 & 232 & 4 & 4 & 4 \\
\hline Canchan & 71 & 24 & 95 & 5 & 69 & 156 & 225 & 5 & 27 & 10 & 37 & 5 & 357 & 167 & 190 & 5 & 5 & 5 \\
\hline CIP387096.2 & 56 & 41 & 97 & 4 & 137 & 189 & 326 & 3 & 95 & 56 & 151 & 1 & 574 & 288 & 286 & 3 & 3 & 3 \\
\hline CIP393079.4 & 154 & 18 & 172 & 1 & 138 & 218 & 356 & 2 & 66 & 64 & 130 & 2 & 658 & 358 & 300 & 2 & 1 & 2 \\
\hline
\end{tabular}

$\mathrm{H}=$ hombres $\mathrm{M}=$ Mujeres $\mathrm{T}=$ Total $\mathrm{R}=$ Ranking

En la región Huancavelica los productores seleccionaron a la cosecha los clones CIP393079.4 y CIP39034.268, en Huánuco los clones CIP387096.2 y CIP393079.4 y en la región Junín los clones CIP396034.268, CIP393079.4 y CIP387096.2, en ese orden de mérito respectivamente. (Tabla 3)

El cultivar Canchan y los clones CIP387096.2 y CIP393079.4, fueron los clones que presentaron la mejor apariencia, sabor y textura en promedio de las regiones Huancavelica, Huánuco y Junín, según los resultados de la prueba organoléptica realizada con los agricultores a la cosecha. Estos resultados confirman la preferencia de los consumidores por la variedad Canchan por su alto contenido de materia seca y buena calidad para fritura en hojuelas y tiras a nivel nacional, pero susceptible a la Rancha y la calidad del clon CIP387096.2 que próximamente será liberada con excelente aptitud para tiras, pero con la ventaja comparativa frente a Canchan de tener resistencia a la Rancha.

En la región Huancavelica los cultivares Canchan y Única fueron las mejores según la prueba organoléptica, y en tercer lugar el clon CIP387096.2. En Junín y Huánuco el cultivar Canchan y los clones CIP387096.2 y CIP393079.4, fueron los mejores en ese orden. Esto nos muestra que la calidad del clon CIP387096.2 que será liberada próximamente como un nuevo cultivar, es muy buena y además es 
resistente a la Rancha, teniendo en cuenta que las cultivares Canchan y Única son muy apreciadas en el mercado por su calidad, pero cuando son sembradas por los agricultores no resisten a la Rancha. (Tabla 4). El Clon CIP386034.268 no presento buenas características organolépticas a pesar de haber sido seleccionada a la cosecha por los agricultores durante la SVP, como uno de los mejores por sus caracteres agronómicos y rendimiento.

Tabla 4. Prueba Organoléptica de los clones y cultivares durante la SVP, para la apariencia, sabor y textura en Huancavelica, Huánuco y Junín. 2016-2017.

\begin{tabular}{ccccccccccccccccc}
\hline \multicolumn{4}{c}{ Huancavelica } & \multicolumn{1}{c}{ Junín } & \multicolumn{1}{c}{ Huanuco } \\
\cline { 2 - 5 } & SBR & TEXT & TOTAL & R & APA & SBR & TEXT & TOTAL & R & APA & SBR & TEXT & TOTAL & R & APA \\
\hline 68 & 115 & 96 & 77 & 288 & $\mathbf{4}$ & 120 & 107 & 93 & 320 & $\mathbf{5}$ & 79 & 77 & 69 & 225 & $\mathbf{4}$ & 314 \\
& 111 & 93 & 327 & $\mathbf{2}$ & 125 & 118 & 89 & 332 & $\mathbf{4}$ & 77 & 81 & 53 & 211 & $\mathbf{5}$ & 325 \\
127 & 103 & 117 & 347 & $\mathbf{1}$ & 151 & 152 & 116 & 419 & $\mathbf{1}$ & 85 & 85 & 77 & 247 & $\mathbf{2}$ & 363 \\
131 & 115 & 79 & 325 & $\mathbf{3}$ & 153 & 144 & 108 & 405 & $\mathbf{2}$ & 91 & 89 & 72 & 252 & $\mathbf{1}$ & 375 \\
113 & 91 & 77 & 281 & $\mathbf{5}$ & 135 & 120 & 91 & 346 & $\mathbf{3}$ & 87 & 85 & 57 & 229 & $\mathbf{3}$ & 335 \\
\hline
\end{tabular}

El análisis de variancia (ANOVA) para la resistencia a la rancha medida a través del Área debajo de la Curva de Progreso de la Enfermedad (AUDPC) (tabla 5), nos muestra que existió diferencias estadísticamente significativas $(\alpha=0.01)$ entre los clones y cultivares en estudio en todas las localidades, debido a las diferencias en sus valores de AUDPC que fueron bajos en todos los clones debido a sus niveles de resistencia a la Rancha, frente a las cultivares susceptibles cuyos valores de AUDPC fueron altos. Los coeficientes de variabilidad (CV) estuvieron dentro de los rangos aceptables, excepto en la localidad de Paca en la región Junín, donde probablemente existió factores climáticos que originaron otras enfermedades fungosas que se confundían con los síntomas de la Rancha y que afectaron de alguna manera al experimento. Según la prueba estadística de comparación de medias de Waller/Duncan $(\alpha=0.05)$ (Tabla 6), los clones CIP387096.2, CIP3930794 y CIP396034.268 fueron estadísticamente iguales en todas las localidades, pero diferentes a los cultivares Canchan y Unica. 
Tabla 5. Análisis de variancia para el Área debajo de la Curva de Progreso de la Enfermedad (AUDPC), en ocho localidades de las regiones de Huancavelica, Huánuco y Junín. 2016-2017.

\begin{tabular}{|c|c|c|c|c|c|c|c|c|c|}
\hline \multirow[b]{3}{*}{ Fuentes de Variacion } & \multirow[b]{3}{*}{ g.l. } & \multicolumn{8}{|c|}{ Cuadrado medio } \\
\hline & & \multicolumn{2}{|c|}{ Huanuco } & \multicolumn{2}{|c|}{ Huancavelica } & \multicolumn{4}{|c|}{ Junin } \\
\hline & & Mayobamba & Quechualoma & Checche & Churcampa & Huasahuasi & Paca & Huarisca & Santa Ana \\
\hline Repeticiones & 2 & 2029.07 ns & 46336.27 ns & $48063.80 \mathrm{~ns}$ & $1363.27 \mathrm{~ns}$ & 140612.87 ns & 9280.27 ns & $11391.80 \mathrm{~ns}$ & 119652.87 ns \\
\hline Clones & 4 & $18174003.77^{* *}$ & $814290.57^{* *}$ & $679531.77^{* *}$ & $439295.17^{* *}$ & $18635380.27 * *$ & 483353.93 ** & 122805.60 ** & $1676301.57 * *$ \\
\hline Error & 8 & 80628.07 & 26173.77 & 19031.47 & 1610.77 & 73378.62 & 23045.68 & 5066.55 & 42088.12 \\
\hline C.V \% & & 12.97 & 29.51 & 27.03 & 12.41 & 14.12 & 39.30 & 31.32 & 28.31 \\
\hline
\end{tabular}

Tabla 6. Prueba de comparación de medias Waller/Duncan $(\alpha=0.01)$ para los valores de AUDPC de los clones y cultivares en estudio en ocho localidades de las regiones de Huancavelica, Huanuco y Junín. 2016-2017

\begin{tabular}{lllllllll}
\hline & \multicolumn{7}{c}{ Huanuco } & \multicolumn{7}{c}{ Huancavelica } \\
\cline { 2 - 9 } Clones & Mayobamba & Quechualoma & Checche & Churcampa & Huasahuasi & Paca & Huarisca & Santa Ana \\
\hline CIP387096.2 & $326 \mathrm{a}$ & $171 \mathrm{a}$ & $165 \mathrm{a}$ & $98 \mathrm{a}$ & $94 \mathrm{a}$ & $89 \mathrm{a}$ & $86 \mathrm{a}$ & $223 \mathrm{a}$ \\
CIP393079.4 & $484 \mathrm{a}$ & $204 \mathrm{a}$ & $183 \mathrm{a}$ & $103 \mathrm{a}$ & $128 \mathrm{a}$ & $103 \mathrm{a}$ & $58 \mathrm{a}$ & $205 \mathrm{a}$ \\
CIP396034.268 & $366 \mathrm{a}$ & $160 \mathrm{a}$ & $188 \mathrm{a}$ & $78 \mathrm{a}$ & $105 \mathrm{a}$ & $94 \mathrm{a}$ & $98 \mathrm{a}$ & $120 \mathrm{a}$ \\
CANCHAN & $4854 \mathrm{~b}$ & $1275 \mathrm{c}$ & $808 \mathrm{~b}$ & $363 \mathrm{~b}$ & $4252 \mathrm{~b}$ & $743 \mathrm{~b}$ & $481 \mathrm{~b}$ & $1425 \mathrm{~b}$ \\
UNICA & $4915 \mathrm{~b}$ & $930 \mathrm{~b}$ & $1208 \mathrm{c}$ & $975 \mathrm{c}$ & $5014 \mathrm{c}$ & $899 \mathrm{~b}$ & $412 \mathrm{~b}$ & $1650 \mathrm{~b}$ \\
\hline
\end{tabular}

Los clones y cultivares con la misma letra son estadísticamente iguales.

El análisis de variancia combinado para cada una de las regiones nos muestra diferencias estadísticamente significativas $(\alpha=0.01)$, para el AUDPC en las fuentes de variación Regiones, clones y en la interacción clones $\mathrm{x}$ regiones, esto indica que los niveles de infección por la rancha en los clones y cultivares tienen diferente magnitud para AUDPC en las tres regiones (Tabla 7).

Tabla 7. Análisis de variancia combinado para el Área debajo de la Curva de Progreso de la Enfermedad (AUDPC), en las regiones de Huancavelica, Huanuco y Junín. 2016-2017.

\begin{tabular}{lcccccc}
\hline & & \multicolumn{3}{c}{ Cuadrado medio } \\
\cline { 3 - 7 } \multicolumn{1}{c}{ Fuentes de Variacion } & g.I. & Huanuco & df & Huancavelica & df & Junin \\
\hline Regiones & 1 & $20193325.63 * *$ & 1 & $261893.63 * *$ & 3 & $8778665.39 * *$ \\
Repeticiones/Regiones & 4 & $24182.67 \mathrm{~ns}$ & 4 & $24713.53 *$ & 8 & $70234.45 *$ \\
Clones & 4 & $13225745.45 * *$ & 4 & $1080961.38 * *$ & 4 & $11040977.98 * *$ \\
Clones x Regiones & 4 & $5762548.88 * *$ & 4 & $37865.55 *$ & 12 & $3292287.80 * *$ \\
Error combinado & 16 & 53400.92 & 16 & 10321.12 & 32 & 35894.70 \\
\hline C.V \% & \multicolumn{7}{c}{16.88} & & 24.37 & & 23.28 \\
\hline
\end{tabular}

La prueba de comparación de medias de Waller/Duncan $(\alpha=0.05)$ nos muestra que los clones CIP387096.2, CIP393079.4 y CIP396034.268, fueron estadísticamente iguales, pero diferentes a los cultivares
Canchan y Unica. En la tabla 8 además podemos ver que por los valores de AUDPC mostrados para los clones son menores que para Única y Canchan en Huanuco y Junín y Huancavelica. En 
Huancavelica los clones fueron menos afectados por Rancha, debido probablemente a la presencia de diferentes razas o poblaciones del patógeno (Phytophthora infestans Mont de Bary) y las condiciones ambientales de lluvia, humedad relativa, temperatura, que si fueron más adecuadas en Huánuco y Junín para tener alta presión de la enfermedad (figura 2).

Tabla 8. Prueba de comparación de medias Waller/Duncan $(\alpha=0.01)$ para los valores de AUDPC de los clones y cultivares en estudio en las regiones de Huancavelica, Huanuco y Junín. 2016-2017

\begin{tabular}{|c|c|c|c|}
\hline Clones & Huánuco & Huancavelica & Junín \\
\hline CIP387096.2 & $249 a$ & $131 \mathrm{a}$ & $123 \mathrm{a}$ \\
\hline CIP393079.4 & $344 \mathrm{a}$ & $143 \mathrm{a}$ & $123 \mathrm{a}$ \\
\hline CIP396034.268 & $263 \mathrm{a}$ & $133 \mathrm{a}$ & $104 \mathrm{a}$ \\
\hline UNICA & $3065 \mathrm{~b}$ & $1091 \mathrm{c}$ & 1994 \\
\hline CANCHAN & $2923 \mathrm{~b}$ & $586 \mathrm{~b}$ & $1725 \mathrm{~b}$ \\
\hline
\end{tabular}

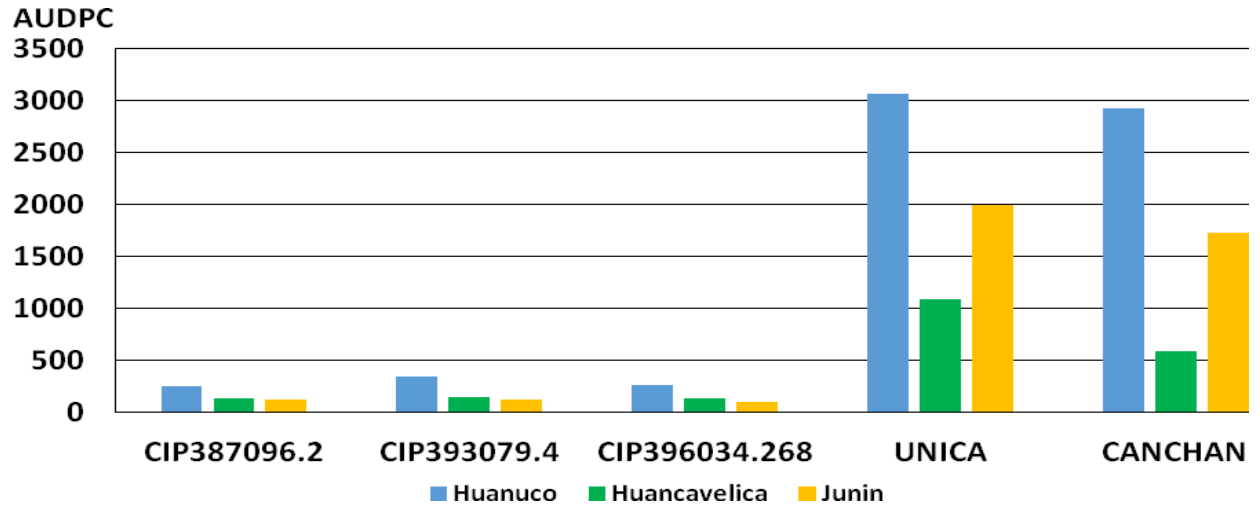

Figura 2. Comportamiento de la resistencia a la Rancha (AUDPC) de los clones en cada una de las 3 regiones 2016 - 2017

El análisis de variancia para rendimiento de tubérculos comerciales por hectárea th 1, nos muestra que existen diferencias estadísticas significativas al $\alpha=0.05$ en todas las localidades menos en
Churcampa y Huasahuasi donde se encontró diferencias estadísticamente diferentes al $\alpha=0.01$ (Tabla 9), El CV estuvo dentro de los rangos adecuados para este tipo de variables en todas las localidades. 
Tabla 9. Análisis de variancia simple para el rendimiento Comercial por hectárea th $^{-1}$, en 8 localidades. 2016 - 2017.

\begin{tabular}{lccccccccc}
\hline & \multicolumn{8}{c}{ Cuadrado medio } \\
\cline { 2 - 10 } & \multicolumn{2}{c}{ Fuentes de Variacion g.l. } & Mayobamba Quechualoma & Checche & Churcampa & Huasahuasi & Paca & Huarisca & Santa Ana \\
\hline Repeticiones & 2 & $2.54 \mathrm{~ns}$ & $0.61 \mathrm{~ns}$ & $74.40^{*}$ & $191.40 \mathrm{~ns}$ & $18.38 \mathrm{~ns}$ & $79.96 \mathrm{~ns}$ & $53.67 \mathrm{~ns}$ & $6.02 \mathrm{~ns}$ \\
Clones & 4 & $16.47^{*}$ & $20.54^{*}$ & $53.35^{*}$ & $942.00^{* *}$ & $317.18^{* *}$ & $104.69 *$ & $151.30^{*}$ & $113.31^{*}$ \\
Error & 8 & 3.67 & 8.67 & 19.16 & 173.80 & 8.84 & 26.68 & 58.46 & 18.53 \\
\hline C.V\% & & 25.94 & 27.81 & 15.70 & 26.10 & 12.79 & 12.41 & 29.57 & 22.76 \\
\hline
\end{tabular}

La prueba de comparación de medias de Waller/Duncan $(\alpha=0.05)$, nos muestra que los rendimientos comerciales por hectárea fueron variables en las 8 localidades, debido probablemente a las características ambientales, suelo, manejo del cultivo diferentes. El clon CIP 396034.268 fue estadísticamente diferente a los demás clones y cultivares en Huasahuasi, en los otras localidades su comportamiento fue variable, En todas las localidades de las regiones de Junín y Huancavelica tuvo el mejor rendimiento que fue desde $28.48 \mathrm{th}^{-1}$ en Santa Ana (Junín) hasta 76.38 th $^{-1}$ en Churcampa (Huancavelica). En las localidades de la región Huanuco, los rendimientos fueron menores que en las otras dos regiones debido probablemente a las condiciones extremas de precipitación debido al cambio climático (Tabla 10).

Tabla 10. Prueba de comparación de medias Waller/Duncan $\alpha=0.05$ para el Rendimiento Comercial en th $^{-1}$ en cada una delas 8 localidades en tha ${ }^{-1}$. 2016-2017

\begin{tabular}{|c|c|c|c|c|c|c|c|c|}
\hline \multirow[b]{2}{*}{ Clones } & \multicolumn{2}{|l|}{ Huanuco } & \multicolumn{2}{|c|}{ Huancavelica } & \multicolumn{4}{|l|}{ Junin } \\
\hline & Mayobamba & Quechualoma & Checche & Churcampa & Huasahuasi & Paca & Huarisca & Santa Ana \\
\hline CIP387096.2 & $9.51 \mathrm{a}$ & $10.75 a b$ & $27.74 a b$ & $31.83 \mathrm{c}$ & $28.18 \mathrm{~b}$ & $36.10 \mathrm{~b}$ & $20.14 a b$ & $17.68 \mathrm{~b}$ \\
\hline CIP393079.4 & 7.55 a & $8.65 a b$ & $27.24 \mathrm{ab}$ & $40.18 b c$ & $27.46 \mathrm{~b}$ & $46.11 a b$ & $29.62 \mathrm{ab}$ & $15.81 \mathrm{~b}$ \\
\hline CIP396034.268 & $8.58 \mathrm{a}$ & $8.00 \mathrm{~b}$ & $34.98 \mathrm{a}$ & $76.38 \mathrm{a}$ & $35.54 \mathrm{a}$ & $49.65 \mathrm{a}$ & $36.37 \mathrm{a}$ & $28.48 \mathrm{a}$ \\
\hline UNICA & 7.88 a & $14.69 \mathrm{a}$ & $24.41 \mathrm{~b}$ & $44.12 b c$ & $12.20 \mathrm{c}$ & $38.65 \mathrm{~b}$ & $19.61 \mathrm{~b}$ & $20.45 a b$ \\
\hline CANCHAN & $3.42 \mathrm{~b}$ & $10.85 a b$ & $25.02 \mathrm{~b}$ & $60.01 \mathrm{ab}$ & $12.86 \mathrm{C}$ & $37.66 \mathrm{~b}$ & $23.54 \mathrm{ab}$ & $12.13 \mathrm{~b}$ \\
\hline
\end{tabular}

El ANOVA combinado en cada región que se muestra en la tabla 11, indica diferencias estadísticamente significativas para las fuentes de variación regiones, clones y clones $\mathrm{x}$ regiones, debido a que existió diferencias en el rendimiento comercial de los clones y cultivares y además estas presentaron interacción con las localidades debido a los diferentes factores ambientales, suelo y manejo del cultivo presente en cada una de las regiones. Los $\mathrm{CV}$ estuvieron dentro de rango aceptable. 
Tabla 11. Análisis de variancia combinado para el Rendimiento Comercial por hectárea th ${ }^{1}$ en las regiones de Huancavelica, Huánuco y Junín. 2016 - 2017.

\begin{tabular}{lcccccc}
\hline & & \multicolumn{7}{c}{ Cuadrado medio } \\
\cline { 3 - 7 } \multicolumn{1}{c}{ Fuentes de Variacion } & g.l. & Huanuco & df & Huancavelica & df & Junin \\
\hline Regiones & 1 & $76.86^{* *}$ & 1 & $3839.75^{* *}$ & 3 & $1471.54^{* *}$ \\
Repeticiones/Regiones & 4 & $1.57 \mathrm{~ns}$ & 4 & $132.90 \mathrm{~ns}$ & 8 & $39.50 \mathrm{~ns}$ \\
Clones & 4 & $16.92^{*}$ & 4 & $638.70^{* *}$ & 4 & $501.95^{* *}$ \\
Clones x Regiones & 4 & $20.09 *$ & 4 & $356.65^{*}$ & 12 & $61.51^{*}$ \\
Error combinado & 16 & 6.17 & 16 & 96.48 & & 28.13 \\
\hline C.V\% & & 27.64 & & 25.06 & & 19.35 \\
\hline
\end{tabular}

La prueba de comparación de medias de Waller/Duncan $(\alpha=0.05)$ nos muestra que existió diferencias de rendimiento de tubérculos comerciales en th ${ }^{-1}$ entre los clones y cultivares. En la región Huanuco los clones CIP387096.2, CIP393079.4 y CIP396034.268 fueron estadísticamente iguales a la variedad Única, pero diferentes a la variedad Canchan, con rendimientos desde $7.14 \mathrm{th}^{-1}$ hasta 11.29 th $^{-1}$. En la región Huancavelica el Clon CIP396034.268 con 55.68 th th $^{-1}$ fue estadísticamente diferente a los demás clones y cultivares, en la región Junín, igualmente este mismo clon con 37.51 fue estadísticamente diferente, seguido por los clones CIP393079.4 con 29.75 th $^{-1} \mathrm{y}$ CIP393079.4 con 25.53 th $^{-1}$ (Tabla 12, figura 3)

Tabla 12. Prueba de comparación de medias Waller/Duncan $\alpha=0.05$ para el Rendimiento Comercial por hectárea th ${ }^{-1}$ en las regiones de Huancavelica, Huánuco y Junín. 2016-2017.

\begin{tabular}{|c|c|c|c|}
\hline Clones & Huánuco & Huancavelica & Junín \\
\hline CIP387096.2 & $10.13 \mathrm{ab}$ & 31.20 & 25.53 \\
\hline CIP393079.4 & $8.10 \mathrm{ab}$ & $33.71 \quad b c$ & $29.75 \mathrm{~b}$ \\
\hline CIP396034.268 & $8.29 \mathrm{ab}$ & $55.68 \mathrm{a}$ & $37.51 \mathrm{a}$ \\
\hline UNICA & $11.29 \mathrm{a}$ & $34.27 \mathrm{bc}$ & 22.73 \\
\hline CANCHAN & $7.14 \quad b$ & $42.51 \mathrm{~b}$ & 21.55 \\
\hline
\end{tabular}




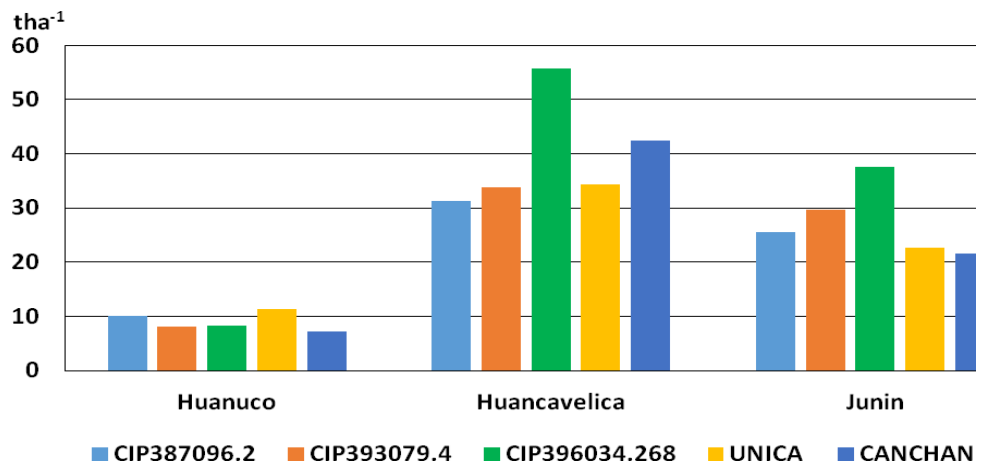

Figura 3. Rendimiento Comercial por hectárea th ${ }^{-1}$ de los clones y cultivares en las regiones de Huancavelica, Huánuco y Junín. 2016 - 2017.

El ANOVA para rendimiento total de tubérculos por hectárea th $^{-1}$, nos muestra que existen diferencias estadísticas significativas al $\alpha=0.05$ en todas las localidades menos en Quechualoma donde se encontró diferencias estadísticamente diferentes al $\alpha=0.01$ (Tabla 13), El CV estuvo dentro de los rangos adecuados para este tipo de variables en todas las localidades.

Tabla 13. Análisis de variancia simple para el Rendimiento Total por hectárea $t^{-1}$, en 8 localidades. 2016 - 2017.

\begin{tabular}{|c|c|c|c|c|c|c|c|c|c|}
\hline \multirow[b]{3}{*}{ Fuentes de Variacion } & & \multicolumn{8}{|c|}{ Cuadrado medio } \\
\hline & & \multicolumn{2}{|c|}{ Huanuco } & \multicolumn{2}{|c|}{ Huancavelica } & \multicolumn{4}{|c|}{ Junin } \\
\hline & gol. & Mayobamba & Quechualoma & Checche & Churcampa & Huasahuasi & Paca & Huarisca & Santa Ana \\
\hline Repeticiones & 2 & $2.25 \mathrm{~ns}$ & $0.54 \mathrm{~ns}$ & $97.78 *$ & 240.45 ns & $7.35 \mathrm{~ns}$ & $74.46 \mathrm{~ns}$ & $72.25 \mathrm{~ns}$ & $6.41 \mathrm{~ns}$ \\
\hline Clones & 4 & $17.10^{*}$ & $16.48 \mathrm{~ns}$ & $57.15^{*}$ & $1026.53^{*}$ & 366.93 ** & $112.83^{*}$ & $166.70 *$ & $115.21 *$ \\
\hline Error & 8 & 3.46 & 12.32 & 14.89 & 225.29 & 13.24 & 32.88 & 61.25 & 18.88 \\
\hline C.V\% & & 23.11 & 30.36 & 12.14 & 27.67 & 14.89 & 13.13 & 27.82 & 21.93 \\
\hline
\end{tabular}

La prueba de comparación de medias de Waller/Duncan $(\alpha=0.05)$, nos muestra que los rendimientos totales por hectárea fueron variables en las 8 localidades, debido probablemente a las características ambientales, suelo, manejo del cultivo diferentes, como se muestra en la tabla
14. En la región Huanuco, los rendimientos fueron menores que en las otras dos regiones debido probablemente a las condiciones extremas de precipitación debido al cambio climático (Tabla 14). 
Tabla 14. Prueba de comparación de medias Waller/Duncan $\alpha=0.05$ para el Rendimiento Total por hectárea th $^{-1}$ en cada una delas 8 localidades

\begin{tabular}{lllllllll}
\hline & \multicolumn{7}{c}{ Huanuco } & \multicolumn{7}{c}{ Huancavelica } \\
\cline { 2 - 9 } \multicolumn{1}{c}{ Clones } & Mayobamba & Quechualoma & Checche & Churcampa & Huasahuasi & Paca & Huarisca & Santa Ana \\
\hline CIP387096.2 & $10.07 \mathrm{a}$ & $11.96 \mathrm{a}$ & $30.57 \mathrm{~b}$ & $34.61 \mathrm{~b}$ & $29.54 \mathrm{a}$ & $38.68 \mathrm{~b}$ & $22.21 \mathrm{ab}$ & $18.62 \mathrm{~b}$ \\
CIP393079.4 & $8.36 \mathrm{a}$ & $10.24 \mathrm{a}$ & $31.72 \mathrm{ab}$ & $44.77 \mathrm{~b}$ & $29.02 \mathrm{a}$ & $48.77 \mathrm{ab}$ & $33.54 \mathrm{ab}$ & $16.69 \mathrm{~b}$ \\
CIP396034.268 & $9.18 \mathrm{a}$ & $8.80 \mathrm{a}$ & $39.24 \mathrm{a}$ & $81.07 \mathrm{a}$ & $37.75 \mathrm{a}$ & $51.72 \mathrm{a}$ & $38.17 \mathrm{a}$ & $29.55 \mathrm{a}$ \\
UNICA & $8.70 \mathrm{a}$ & $15.09 \mathrm{a}$ & $28.90 \mathrm{~b}$ & $46.16 \mathrm{~b}$ & $12.61 \mathrm{c}$ & $40.32 \mathrm{ab}$ & $20.95 \mathrm{~b}$ & $21.15 \mathrm{ab}$ \\
CANCHAN & $3.93 \mathrm{~b}$ & $11.71 \mathrm{a}$ & $28.50 \mathrm{~b}$ & $64.66 \mathrm{ab}$ & $13.25 \mathrm{c}$ & $38.84 \mathrm{~b}$ & $25.76 \mathrm{ab}$ & $13.04 \mathrm{~b}$ \\
\hline
\end{tabular}

El análisis de variancia combinado en cada región que se muestra en la tabla 15 , indica diferencias estadísticamente significativas en Huancavelica y Junín, para las fuentes de variación regiones, clones y clones $\mathrm{x}$ regiones, debido a que existió diferencias en el rendimiento total de los clones y cultivares y además estas presentaron interacción con las localidades debido a los diferentes factores ambientales, suelo y manejo del cultivo presente en cada una de las regiones. En la región Huanuco no se encontró significación para Clones y la interacción clones $\mathrm{x}$ regiones. Los $\mathrm{CV}$ estuvieron dentro del rango aceptable.

Tabla 15. Análisis de variancia combinado para el Rendimiento Total por hectárea $t^{-1}$ en las regiones de Huancavelica, Huánuco y Junín. 2016 - 2017.

\begin{tabular}{lcccccc}
\hline & & \multicolumn{3}{c}{ Cuadrado medio } & & \\
\cline { 3 - 7 } \multicolumn{1}{c}{ Fuentes de Variacion } & g.l. & Huanuco & df & Huancavelica & df & Junin \\
\hline Regiones & 1 & $92.65 * *$ & 1 & $3784.73^{* *}$ & 3 & $1605.68 * *$ \\
Repeticiones/ Regiones & 4 & $1.40 \mathrm{~ns}$ & 4 & $167.61 \mathrm{~ns}$ & 8 & $40.12 \mathrm{~ns}$ \\
Clones & 4 & $16.04 \mathrm{~ns}$ & 4 & $707.60 * *$ & 4 & $554.99 * *$ \\
Clones x Regiones & 4 & $17.55 \mathrm{~ns}$ & 4 & $376.08 *$ & 12 & $68.90^{*}$ \\
Error combinado & 16 & 7.89 & 16 & 120.09 & & 31.56 \\
\hline C.V \% & & 28.65 & & 25.47 & & 19.37 \\
\hline
\end{tabular}

La prueba de comparación de medias de Waller/Duncan $(\alpha=0.05)$ nos muestra que existió diferencias de rendimiento de tubérculos totales en th ${ }^{-1}$ entre los clones y cultivares en las regiones de Huancavelica y Junín. En la región Huanuco los clones CIP387096.2, CIP393079.4 y CIP396034.268 fueron estadísticamente iguales a los cultivares Única y Canchan, con rendimientos desde 7.82 th $^{-1}$ hasta 11.90 th $^{-1}$. En la región Huancavelica el Clon CIP396034.268 con 60.16 th $^{-1}$ fue estadísticamente diferente a los demás clones y cultivares, en la región Junín, igualmente este mismo clon con 39.30 fue estadísticamente diferente, seguido por los clones CIP393079.4 con 29.75 th $^{-1}$ y CIP393079.4 con 25.53 th $^{-1}$ (Tabla 16, figura 4) 
Tabla 16. Prueba de comparación de medias Waller/Duncan $\alpha=0.05$ para el Rendimiento Total por hectárea th $^{-1}$ en las regiones de Huancavelica, Huánuco y Junín. 2016-2017.

\begin{tabular}{llllll}
\hline Clones & Huánuco & Huancavelica & Junín & \\
\hline CIP387096.2 & $11.02 \mathrm{a}$ & $32.59 \mathrm{c}$ & 27.26 & $\mathrm{c}$ \\
CIP393079.4 & $9.30 \mathrm{a}$ & $38.25 \mathrm{bc}$ & $32.01 \mathrm{~b}$ & \\
CIP396034.268 & $8.99 \mathrm{a}$ & $60.16 \mathrm{a}$ & $39.30 \mathrm{a}$ & \\
UNICA & $11.90 \mathrm{a}$ & $37.33 \mathrm{bc}$ & 23.76 & $\mathrm{~cd}$ \\
CANCHAN & $7.82 \mathrm{a}$ & $46.78 \mathrm{ab}$ & 22.72 & $\mathrm{~d}$ \\
\hline
\end{tabular}

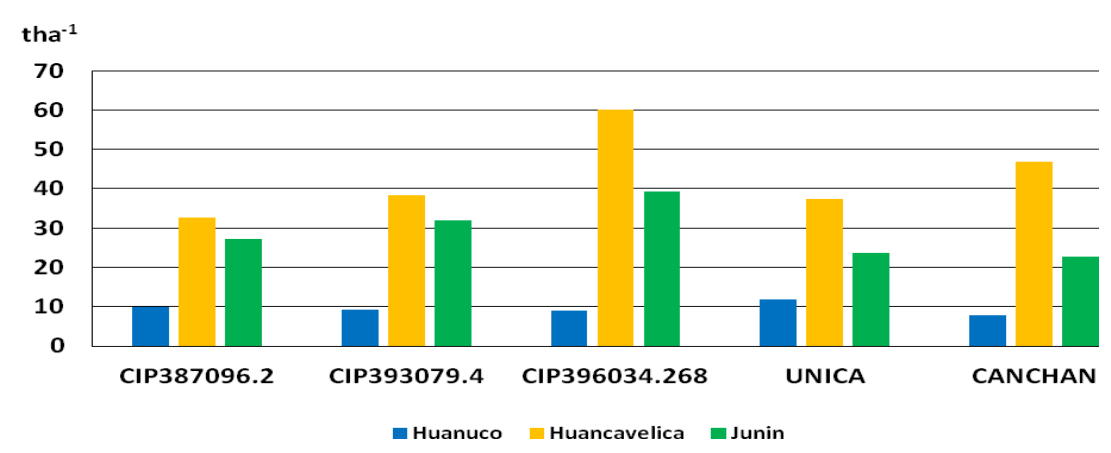

Figura 4. Rendimiento Total por hectárea th $^{-1}$ de los clones y cultivares en cada una de las 3 regiones: Huancavelica, Huánuco, Junín 2016-2017.

\section{Agradecimientos}

Ninguno.

\section{Conflictos de intereses}

El presente documento no presenta conflictos de intereses.

\section{Referencias citadas}

Allauca, S. (2011). Análisis de los criterios de preferencias en la selección de nuevas variedades de papa, con enfoque en la influencia del mercado. Tesis para optar el grado académico de Magister Scientiae en Producción y Extensión Agrícola. Universidad Nacional Agraria, La Molina, Lima, Perú. 229 p.

Arcos, J.; Gastelo, M.; Holguin, V. (2015). INIA 317 - Altiplano, variedad de papa con buena adaptación en la región altiplánica del Perú. Revista Latinoamericana de la Papa 19 (2): 68-75

Cuesta, X.; Andrade, H. (2001).El Mejoramiento participativo de papa en el Ecuador. INIAP-PNRT-papa. PREDUZA Quito Ecuador.

De Haan, S.; Salas, E.; Fonseca, C.; Gastelo, M.; Amaya, N.; Bastos, C.; Hualla, V.; Bonierbale, M. (2017). Selección participativa de cultivares de papa (SPV) usando el diseño mama y bebe: una guía para capacitadores con perspectiva de género. Lima (Perú). Centro Internacional de la Papa. 82 pp.

Egusquiza. R. (2000). La Papa. Producción, Transformación y 
Comercialización. Universidad Agraria La Molina. Convenio ADEX-AID/MSP. Lima, Perú.192 p

Fierro, L.; Moreno, J. (1999). La Investigación Participativa en el Proceso de selección de cultivares de papa.

Gabriel, J.; Herbas, J.; Salazar, M.; Thiele, G. (2002). Manual Técnico de Mejoramiento Participativo para Obtener nuevas Variedades de Papa. Promoción e Investigación de Productos Andinos, Proyecto de Mejoramiento Participativo. PROINPA. Bolivia.

Galceran, P. (2015). Selección participativa bajo el diseño mamá $\&$ bebé de 4 clones avanzados de papa Solanum tuberosum spp andígena (Población B1C5), para obtener nuevas variedades de papa, en el distrito de Anchonga, del departamento de Huancavelica. Tesis para Ingeniero Agrónomo. Universidad Nacional del Centro, Huancayo, Perú. 124 pág.

Hellin, J.; Bellon M.; Badstue L. (2010). Reduciendo la brecha entre la realidad de los investigadores y la de los agricultores. LEISA Revista de Agroecológica. Diciembre 2010 22:3. Lima Perú.

Janampa, A. (2012). Selección participativa bajo el diseño mamá \& bebé de 20 clones de papa Solanum tuberosum spp. andígena (población B1C5), con resistencia horizontal a la rancha (Phytophthora infestans). Tesis Ingeniero agrónomo. Universidad para el Desarrollo Andino (UDEA), Huancavelica, Perú. 127 pág.

Joshi, A.; Witcombe, J. (1996). Farmer Participatory Crop Improvement. II. Participatory Varietal Selection, a Case Study in India. Experimental Agriculture, 32(4), 461-477. doi: $10.1017 / \mathrm{S} 0014479700001538$
Landeo, J.A.; Gastelo, M.A.; Pacheco, M.A.; De Haan, S.; Diaz, L.; Puente de la Vega, E.; Comunidad campesina. (2008). Two new potato varieties (Solanum tuberosum ssp. andigena) with horizontal resistance to late blight selected by Andean communities through participatory variety selection. Poster presented at: Potato science for the poor; challenges for the new millenium. A working conference to celebrate the International year of the Potato, Cusco, Peru 25-28 March.

Lilja, N.; Ashby J. A. (1999). Types of participatory research based on locus of decision making. CGIAR Systemwide Program on Participatory Research and Gender Analysis. Working document, No. 6. Cali, Colombia. 8p

Mudege N.N.; Mukewa, E.; Amele A. (2015). Workshop Report: Training on Gender Integrated Potato Participatory Varietal Selection (PVS) in Ethiopia. Addis Ababa, Ethiopia, CIP. 26 p.

Ortiz, O. (Editor). 2002. Introducción a la Investigación Participativa. Módulos de Capacitación. Morris, M.L. and M.R. Bellon. 2004. Participatory plant breeding research: Opportunities and challenges for the international crop improvement system. Euphytica 136:21-35. Centro Internacional de la Papa. 45 p.

Semagn A. K.; $\quad$ Donald H.; $\quad$ Keith P.; Walter D. J.; Fentahun M. T.; David W. (2105) Identification of Farmer Priorities in Potato Production Through Participatory Variety Selection. American Journal of Potato Research Volume 92, pp 648-661

Snapp, S. (2002). Quantifying farmer evaluation of technologies: The mother and baby trial design. In: Bellon, M.R., and J. Reeves,(eds.). Quantitative Analysis of Data from participatory 
Methods of Plant Breeding. pp. 10-17. CIMMYT: Mexico, DF.

Tesfaye Tadesse Tefera (2013). Participatory variety selection of Potato (Solanium tuberosum L) in Southern Ethiopia. Journal of Agri-Food and Applied Sciences. On line at jaas.blueap.org (C2013 JAAS Journal. Vol. 1(1), pp. 1-4, 5 .

Thomas S.W. (2008). Participatory Varietal Selection, Participatory Plant Breeding, and Varietal Change. WORLD DEVELOPMENT REPORT 2008
Wangchuk, P.; De Haan, S.; Dochen, R. (2015). Participatory Varietal Selection using Mother and Baby Trial Design in Potato. Department of Agriculture Ministry of Agriculture and Forests, Thimpu, Bhutan.

Witcombe, J.R., A. Joshi, K.D. Joshi, and B.R. Sthapit. (1996). Farmer participatory crop improvement. 1. Varietal selection and breeding methods and their impact on biodiversity. Expl. Agric. 32(4):445-460. 